\title{
Analysis of Thermal Diffusivity of Metals using Lock-in Thermography
}

\author{
by F. Wagner*, T. Malvisalo*, P. W. Nolte**, and S. Schweizer** \\ * Department of Electrical Engineering, South Westphalia University of Applied Sciences, Luebecker Ring 2, \\ 59494 Soest, Germany, wagner.florian@fh-swf.de \\ ${ }^{* *}$ Fraunhofer Application Center for Inorganic Phosphors, Branch Lab of Fraunhofer Institute for Microstructure \\ of Materials and Systems IMWS, Luebecker Ring 2, 59494 Soest, Germany, peter.nolte@imws.fraunhofer.de
}

\begin{abstract}
The thermal diffusivity, $\sigma$, is determined for sheets of different metals by means of an approach based on lock-in thermography in combination with periodical laser heating. Different lock-in frequencies are used to obtain the frequencydependent phase behavior of thermal waves generated by laser pulses. A subsequent curve fitting provides the coefficients required to determine the thermal diffusivity. To evaluate the effect of ambient conditions, the measurements are also performed in vacuum. A comparison with literature data substantiates that lock-in infrared thermography is a promising approach for the determination of the thermal diffusivity.
\end{abstract}

\section{Introduction}

An accurate knowledge of thermal material properties is important for various technical applications. The thermal diffusivity, $\sigma$, is a complex material parameter which depends on the thermal conductivity, $k$, the specific heat capacity, $c_{p}$, as well as the mass density, $\rho$. The infrared (IR) lock-in thermography approach is based on the generation of thermal waves in the material under investigation. The thermal response is then recorded by an IR camera and is analyzed for the amplitude and phase information of the thermal waves.

This work focuses on the analysis of the thermal diffusivity of different metal samples of various thicknesses under periodical heating by laser pulses. Contact-free and non-destructive (active) IR lock-in thermography enables the detection of the amplitude and phase information by monitoring the temporal development of the surface temperature [1]. The measured phase data and lock-in frequency are fitted based on the three-dimensional heat equation and thereon, the thermal diffusivity is determined. A comparison between measurements under ambient conditions and in vacuum is also carried out.

\section{Experimental details}

The metal samples under study are sheets of brass (CuZn37 / Ms63), stainless steel (AISI304 / EN1.4301) and structural steel (St1403 / EN1.0338), each with dimensions of $75 \mathrm{~mm} \times 100 \mathrm{~mm}$ and different thicknesses of $0.5 \mathrm{~mm}$, $0.8 \mathrm{~mm}, 1.0 \mathrm{~mm}$, and $1.5 \mathrm{~mm}$. An overview of the investigated samples is given in Table 1. To maximize optical absorption and to enable a uniform emissivity, the sample surfaces are painted black on both sides.

Table 1. Materials and thicknesses of the investigated samples.

\begin{tabular}{|l|l|}
\hline Samples & thickness / mm \\
\hline brass (CuZn37 / Ms63) & $0.5,0.8,1.0$ and 1.5 \\
\hline stainless steel (AISI304 / EN1.4301) & $0.5,0.8,1.0$ and 1.5 \\
\hline structural steel (St1403 / EN1.0338) & 1.0 and 1.5 \\
\hline
\end{tabular}

For the analysis of thermal diffusivities, the setup consists of an IR camera, a laser diode for optical heating, a sample holder and a lock-in synchronization box, as shown in Fig. 1(a). The entire setup is placed in a closed sample chamber, entirely in black, to screen it from thermal radiation from the external environment. To evaluate the effect of convection, the measurements are also performed in a closed vacuum chamber, see Fig. 1(b). This chamber includes two glass windows: a sapphire glass that is transparent for IR radiation and a borosilicate glass transparent for the laser wavelength of $980 \mathrm{~nm}$. For the measurements, the chamber is evacuated to pressures below 10 mbar. The IR camera (InfraTec ImageIR 8380S) uses an indium antimonide (InSb) focal plane array (FPA) snapshot detector with a geometric resolution of $640 \times 512 \mathrm{px}^{2}$; the spectral range for detection is between $2.0 \mu \mathrm{m}$ and $5.7 \mu \mathrm{m}$. 

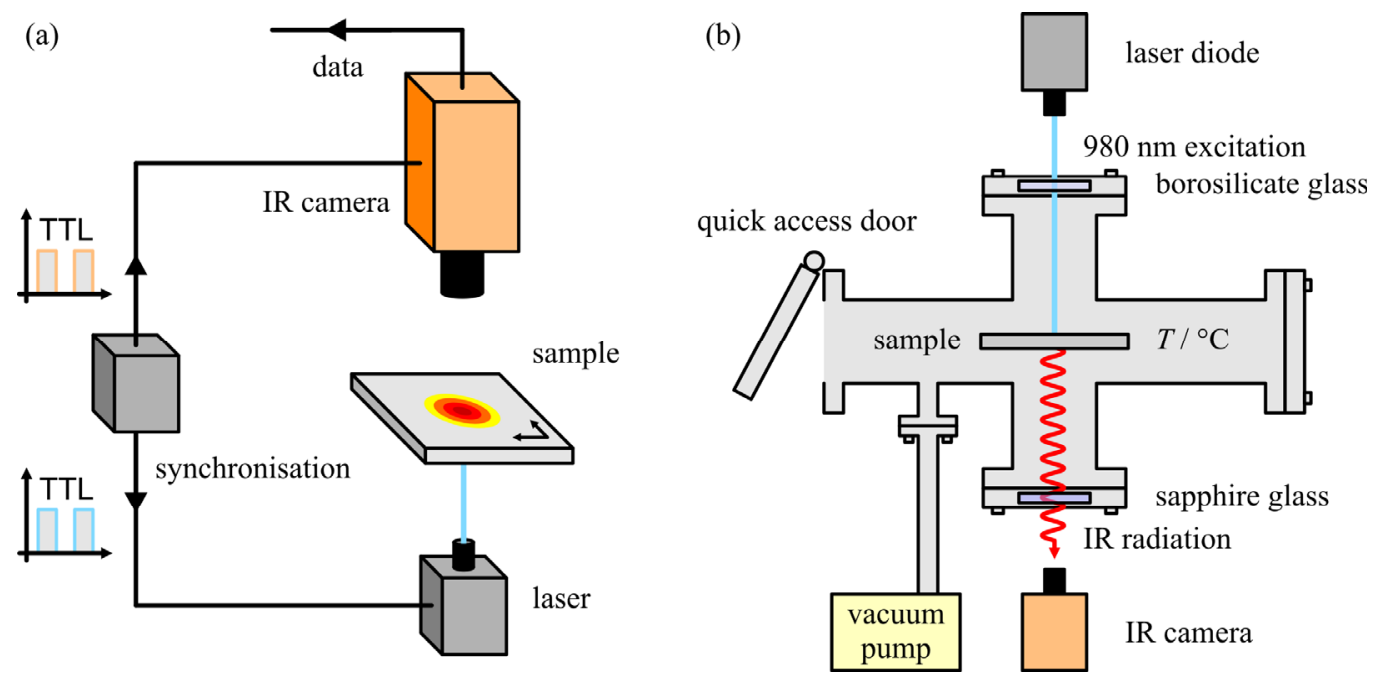

Fig. 1. Lock-in thermography setup to measure the amplitude and phase information of thermal waves (a) under ambient conditions and (b) in vacuum. The laser operates at a wavelength of $980 \mathrm{~nm}$ and has an optical output power of $100 \mathrm{~mW}$. The sample surface is coated in black to increase optical absorption.

For lock-in thermography measurements, the laser is positioned directly below the sample, while the IR camera lens is placed $20 \mathrm{~cm}$ from the sample. The excitation laser with a power of $100 \mathrm{~mW}$ at $980 \mathrm{~nm}$ is directly focused to the center of the sample. The laser and the IR camera are connected to the lock-in box which synchronizes the image capturing with the laser pulses at a certain lock-in frequency, $f$. The modulated laser beam generates thermal waves in the material. The surface temperature, $T$, the amplitude, $A$, as well as the phase delay, $\phi$, are recorded at the backside of the sample. Assuming a harmonic heat generation, the system can be described by the non-steady-state heat equation:

$$
\frac{1}{\sigma} \frac{\mathrm{d}}{\mathrm{d} t} T-\Delta T=\frac{g}{k}
$$

where $k$ is the thermal conductivity, $g$ the volumetric heat rate and $\sigma$ the thermal diffusivity which is defined as:

$$
\sigma=\frac{k}{\rho \cdot c_{\mathrm{p}}}
$$

with $\rho$ the mass density and $c_{p}$ the specific heat capacity.

To calculate the temperature distribution, the three-dimensional heat equation needs to be solved by assuming a harmonically oscillating point source in spherical coordinates. This leads to [1]:

$$
T(r, t)=A \cdot \exp \left(\frac{-r}{\sqrt{\sigma / \pi \cdot f}}\right) \cdot \exp \left(\mathbf{i}\left(\omega t-\frac{r}{\sqrt{\sigma / \pi \cdot f}}\right)\right)
$$

The phase delay of the thermal wave, $\phi_{\text {wave, }}$ can be expressed as

$$
\phi_{\text {wave }}=\frac{r}{\sqrt{\sigma /(\pi \cdot f)}}
$$

The total phase delay, $\phi$, obtained from lock-in measurements, consists beside the phase of the heat wave, $\phi_{\text {wave, }}$ also of a system-depending phase delay, $\phi_{\text {system }}$.

$$
\phi=\phi_{\text {system }}-\phi_{\text {wave }}=\phi_{\text {system }}-\frac{r}{\sqrt{\sigma /(\pi \cdot f)}}
$$

Introducing the variable $\alpha$, which contains the thermal diffusivity, $\sigma$, as well as the penetration depth, $r$, is given by 


$$
\alpha=r \cdot \sqrt{\frac{\pi}{\sigma}}
$$

Eventually, the phase delay, $\phi$, of the thermal wave can be expressed as follows:

$$
\phi=\phi_{\text {system }}-r \cdot \sqrt{\frac{\pi}{\sigma}} \cdot \sqrt{f}=\phi_{\text {system }}-\alpha \cdot \sqrt{f}
$$

For each sample, the lock-in frequency, $f$, is varied within a specific range and the corresponding phase delay is subsequently plotted versus the lock-in frequency. To determine the thermal diffusivity, $\sigma$, the measured phase delay is analyzed on the basis of Eq. (6). The obtained fitting parameter, $\alpha$, is then used to calculate the thermal diffusivity, $\sigma$.

\section{Results and Discussion}

\subsection{Lock-in thermography}

To determine the thermal diffusivity, $\sigma$, the amplitude and phase information of the generated thermal waves are measured. To ensure reproducibility of measurements, an initial warm-up time of several minutes is necessary to allow for the temperature, amplitude and phase information to stabilize. For each sample, a frequency range between $0.25 \mathrm{~Hz}$ to $49.5 \mathrm{~Hz}$ is set. The time between measurements at different frequencies was kept as short as possible to avoid cooling of the sample. Fig. 2(a) shows a thermographic image of the stainless steel sample (EN1.4301) after several minutes of laser heating with a lock-in frequency of $1.0 \mathrm{~Hz}$. Due to the increase of the temperature resolution by lock-in thermography, Fig. 2(b) shows the amplitude of the thermal waves. For the analysis of the thermal diffusivity, the phase information as shown in Fig. 2(c) is directly recorded at the location of the maximum amplitude.
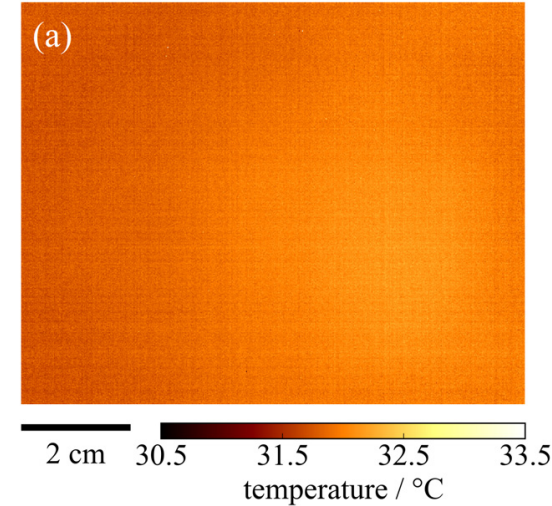

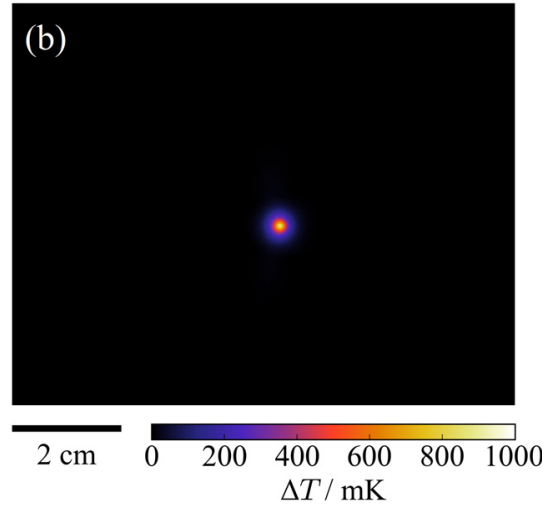

Fig. 2. (a) Thermographic image, (b) amplitude, and (c) phase information of stainless steel (EN1.4301) after several minutes of laser heating at a lock-in frequency of $1.0 \mathrm{~Hz}$. The phase information is an essential parameter to determine the thermal diffusivity.

\subsection{Analysis of the thermal diffusivity under ambient conditions}

Figures 3(a) to 3(c) show the experimentally determined phase delays (scatter) along with their corresponding fitting curves (solid lines) for three different materials, namely brass, stainless steel and structural steel. Due to the detection limit of the IR camera, only phase delays obtained for amplitude signals larger than $10 \mathrm{mK}$, can be precisely determined. Fig. 3(d) depicts the comparison between the calculated thermal diffusivities, $\sigma$, and their literature counterparts for the three different materials. These results are also summarized in Table 2. 
10.21611/qirt.2016.093

Table 2. Comparison of calculated thermal diffusivity values for different materials to literature data.

\begin{tabular}{|l|l|l|l|}
\hline Samples & thickness / mm & \multicolumn{2}{|l|}{ thermal diffusivity $/ \mathbf{1 0}^{-\mathbf{6}} \mathbf{~}^{2} \cdot \mathbf{s}^{-1}$} \\
\cline { 3 - 4 } & & experiment & literature \\
\hline brass (CuZn37 / Ms63) & 0.5 & 34 & $36.5[2]$ \\
& 0.8 & 40 & \\
& 1.0 & 40 & \\
& 1.5 & 41 & $3.8[3]$ \\
\hline stainless steel (AISI304 / EN1.4301) & 0.5 & 3.6 & \\
& 0.8 & 3.9 & \\
\hline structural steel (St1403 / EN1.0338) & 1.0 & 4.0 & \\
& 1.5 & 4.1 & $17.1[4]$ \\
\hline
\end{tabular}
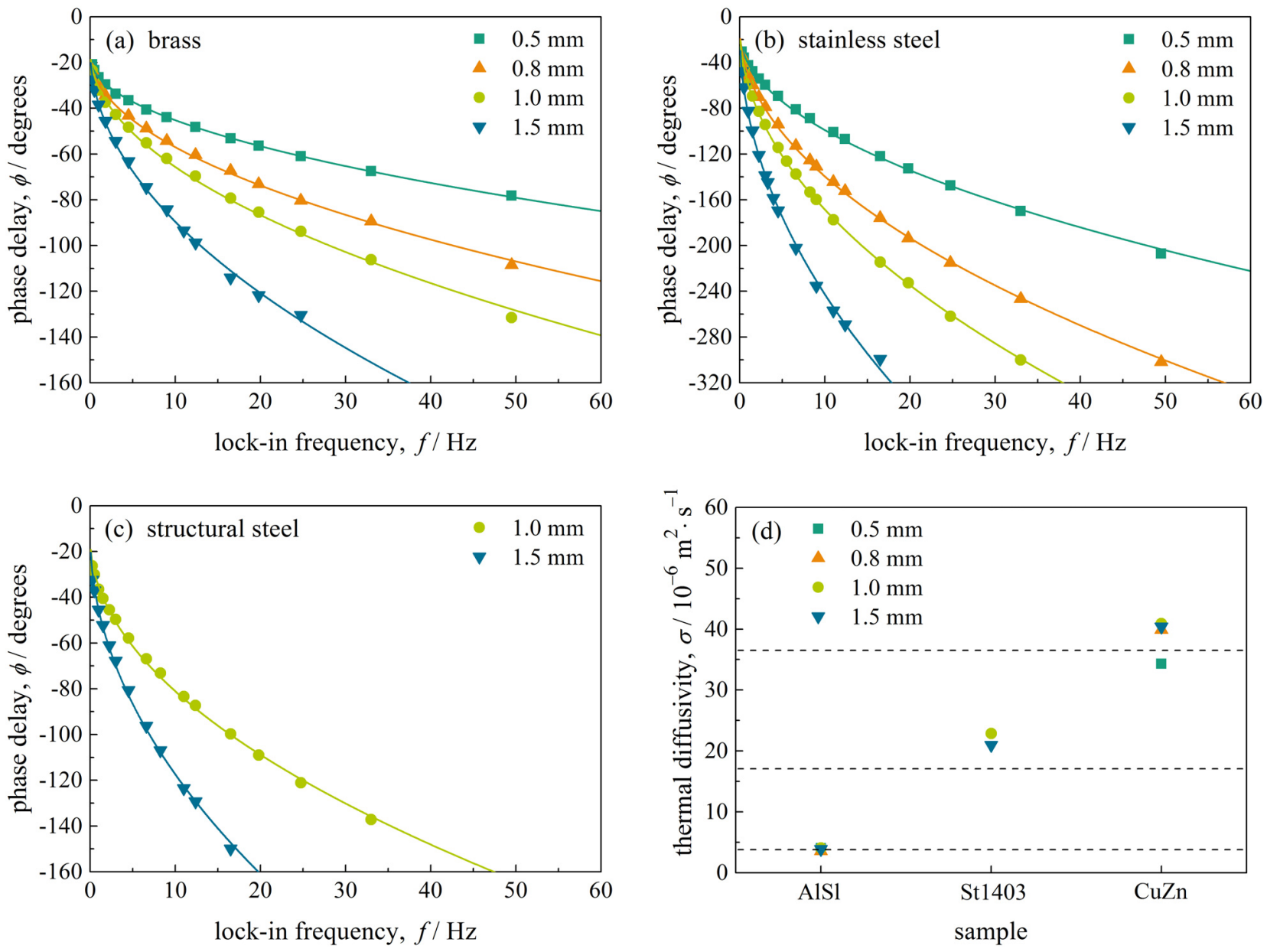

Fig. 3. (a) to (c) experimental phase delays (scatter) and the corresponding fitting curves (solid lines) and (d) calculated thermal diffusivities for different materials and thicknesses and corresponding literature values (dashed lines). 


\subsection{1/qirt.2016.093}

\subsection{Measurements in a vacuum chamber}

Fig. 4(a) shows the experimental phase delays (scatter) in vacuum and the corresponding fitting curves (solid lines) for stainless steel. Due to the high absorption of the windows installed in the vacuum chamber, only the stainless steel sample shows reliable amplitude and phase values. Fig. 4(b) depicts the calculated thermal diffusivities, $\sigma$, for different thicknesses of stainless steel. The results are as summarized in Table 3.
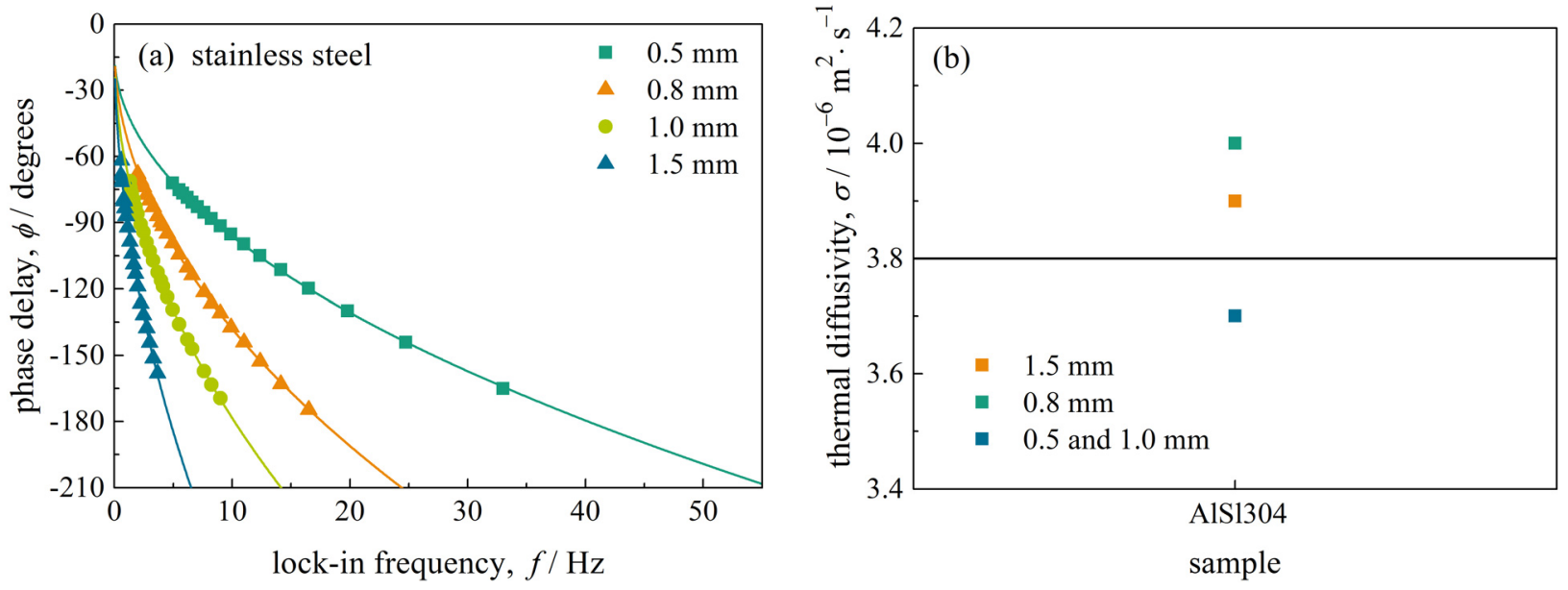

Fig. 4. (a) Experimental phase delays (scatter) in vacuum and the corresponding fitting curves (solid lines), (b) calculated thermal diffusivities, $\sigma$, for stainless steel.

Table 3. Calculated thermal diffusivity values for stainless steel in vacuum, compared to literature data.

\begin{tabular}{|l|l|l|c|}
\hline samples & thickness $/ \mathbf{~ m m}$ & \multicolumn{2}{|l|}{ thermal diffusivity $/ \mathbf{1 0}^{-6} \mathbf{~}^{2} \cdot \mathbf{s}^{-1}$} \\
\cline { 3 - 4 } & & experiment & Literature \\
\hline stainless steel (AISI304 / EN1.4301) & 0.5 & 3.7 & $3.8[2]$ \\
& 0.8 & 4.0 & \\
& 1.0 & 3.7 & \\
\hline
\end{tabular}

\section{Conclusion}

Comparison between experimental results and corresponding literature data demonstrates the promising potential of the proposed lock-in thermography approach to determine the thermal diffusivity by fitting the experimentallydetermined phase. The fitting curves follow the trend of the experimental values and the calculated values for the thermal diffusivity are in good agreement with their corresponding literature data. Comparison of experimentally determined thermal conductivity values of Tables 2 and 3 show that carrying the measurements in vacuum do not improve the accuracy of the thermal diffusivity values. Moreover, it is noteworthy that measurements in vacuum are challenging due to the reduction of the incident laser power and the infrared radiation at the windows of the vacuum chamber, entailing a decrease in signal-to-noise ratio. With increasing thermal conductivity and material thickness, the experimental error in the measurements grows larger due to this reduced signal-to-noise ratio. Increasing the laser power represents a potential solution for better signal-to-noise ratio and thus smaller experimental error.

\section{Acknowledgements}

The authors would like to thank the "Ministerium für Innovation, Wissenschaft und Forschung des Landes Nordrhein-Westfalen" for its financial support to the Fraunhofer Application Center for Inorganic Phosphors in Soest as well as to the South Westphalia University of Applied Sciences within the FH STRUKTUR 2014 project "LED-2020". 
10.21611/qirt.2016.093

\section{REFERENCES}

[1] O. Breitenstein, W. Warta, M. Langenkamp, Lock-in Thermography - Basics and Use for Evaluating Electronic Materials and Devices, second ed., Springer, Berlin/Heidelberg, 2010.

[2] Technical Datasheet - CuZn37, Rev. 13, Aurubis, 2005.

[3] Technical Datasheet - Stainless Steel 1.4301, Rev. 4301-1. Deutsche Edelstahlwerke. 2007.

[4] R. B. Ross, Metallic Materials Specification Handbook, fourth ed. Springer, 1991. 